\title{
Efficient modeling techniques for plasmonic and photonic devices
}

\author{
Amgad Abdrabou ${ }^{1}$ and S. S. A. Obayya ${ }^{2, *}$ \\ ${ }^{1}$ Department of Mathematics, City University of Hong Kong, Kowloon, Hong Kong, China \\ ${ }^{2}$ Center for Photonics and Smart Materials, Zewail City of Science and Technology, Giza, 12578, Egypt
}

\begin{abstract}
Plasmonics plays a vital role in realizing nanophotonic devices for integrated optics due to its strong light localization into subwavelength dimensions beyond the diffraction limit. Therefore, plasmonics has a wide range of applications such as sensing, solar cells, microscopy, etc. Plasmonics modelling techniques are necessary for understanding the underlying physics of plasmonic devices. However, correct modelling of these devices is still an obstacle facing some of existing modeling techniques. In this paper, we discuss the shortcomings of the existing tools for analysing plasmonic devices, highlight their main features and suggest some powerful remedies.
\end{abstract}

\section{Introduction}

Nowadays, plasmonics is playing a vital role in the realizing nanophotonic devices for integrated optics, where light can be confined into subwavelength dimensions beyond the diffraction limit [1]. This featured plasmoincs in a wide range of applications such as sensing, solar cells, etc [1-3]. To understand the underlying physics of plasmonics and for design purposes, correct modelling techniques are essential tools. Unfortunately, many of the conventional techniques either fail [4], or show incurable instabilities [5, 6] due to strong discontinuities at metaldielectric interfaces and the inherent difficulties in imposing the interface boundary conditions. Many trials have been made to overcome such problem by developing efficient and accurate tools specifically for plasmonics [6-9].

In this paper, we introduce an efficient method based on the pseudo-spectral method [10-12], for plasmonic modelling with a proper application of physical boundary conditions at interfaces of discontinuity. The pseudospectral method can incorporate maps which redefine the basis functions over a desired domain.

\section{Mathematical Formulation}

For multi-layered structures invariant along both $x$ and $z$ directions as shown in figure 1, the 1D Helmholtz equation for TE polarization is given by

$$
\frac{\partial^{2} u}{\partial y^{2}}+k_{0}^{2} \varepsilon(y) u=\beta^{2} u, \quad y \in(-\infty, \infty)
$$

while for the TM case we have

$$
\varepsilon(y) \frac{\partial}{\partial y}\left[\frac{1}{\varepsilon(y)} \frac{\partial \phi}{\partial y}\right]+k_{0}^{2} \varepsilon(y) u=\beta^{2} u, \quad y \in(-\infty, \infty)
$$

where $E_{x}=u(y) e^{i \beta z}$ for the TE case, and $H_{x}=u(y) e^{i \beta z}$ for the TM case, $k_{0}$ is the freespace wavenumber and $\beta$ is the propagation constant.

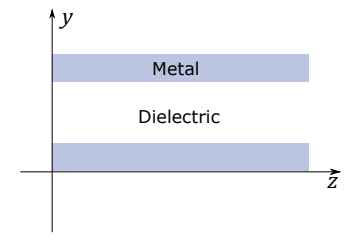

Figure 1. Schematic for a typical multi-layered plasmonic structure.

\section{Multi-domain pseudo-spectra method}

The eigenvalue problems (1) for TE and (2) for TM polarization can be solved numerically using the Chebyshev pseudo-spectral method [10, 19]. By referring to Fig. 1, we can assume that the points of discontinuity of $\varepsilon(y)$ are located at $0=y_{0}<y_{1}<y_{2}<\cdots<y_{N}=b$, where $b>0$. For $s=1,2, \cdots, N$, the $s$ th-subdomain is defined by $y_{s-1}<y<y_{s}$. The Chebyshev collocation points discretize $y$ on each subdomain using $N_{s}+1$ points as

$$
\xi_{s, k}=y_{s-1}+\frac{y_{s}-y_{s-1}}{2}\left[1-\cos \left(k \pi / N_{s}\right)\right], \quad 0 \leq k \leq N_{s} .
$$

Over each subdomain $s$, the method approximates the first derivative of $u(y)$ by a differentiation matrix $\boldsymbol{D}^{(s)}[10]$ such that

$$
\left[\begin{array}{c}
u^{\prime}\left(y_{s-1}^{+}\right) \\
\boldsymbol{u}_{s}^{\prime} \\
u^{\prime}\left(y_{s}^{-}\right)
\end{array}\right] \approx \boldsymbol{D}^{(s)}\left[\begin{array}{c}
u\left(y_{s-1}^{+}\right) \\
\mathbf{u}_{s} \\
u\left(y_{s}^{-}\right)
\end{array}\right]
$$

where $\boldsymbol{u}_{s}$ is a column vector of length $N_{s}-1$ whose entries are $u$ at the interior discretization points, i.e.,

$$
\boldsymbol{u}_{s}=\left[u\left(\xi_{s, 1}\right), u\left(\xi_{s, 2}\right), \cdots, u\left(\xi_{s, N_{s}-1}\right)\right]^{T},
$$


the prime denotes the derivative with respect to $y$. The matrix $\boldsymbol{D}^{(s)}$ can be partitioned as

$$
\boldsymbol{D}^{(s)}=\left[\begin{array}{c|c|c}
d_{1}^{(s)} & \mathbf{d}_{2}^{(s)} & d_{3}^{(s)} \\
\hline \mathbf{d}_{4}^{(s)} & \mathbf{D}_{5}^{(s)} & \mathbf{d}_{6}^{(s)} \\
\hline d_{7}^{(s)} & \mathbf{d}_{8}^{(s)} & d_{9}^{(s)}
\end{array}\right] .
$$

This leads to the approximations [19]

$$
\begin{aligned}
u^{\prime}\left(y_{s-1}^{+}\right) & =d_{1}^{(s)} u\left(y_{s-1}\right)+\mathbf{d}_{2}^{(s)} \boldsymbol{u}_{s}+d_{3}^{(s)} u\left(y_{s}\right), \\
u^{\prime}\left(y_{s}^{-}\right) & =d_{7}^{(s)} u\left(y_{s-1}\right)+\mathbf{d}_{8}^{(s)} \boldsymbol{u}_{s}+d_{9}^{(s)} u\left(y_{s}\right),
\end{aligned}
$$

for the derivatives at the points of discontinuity, and

$$
\boldsymbol{u}^{\prime}=\mathbf{d}_{4}^{(s)} u\left(y_{s-1}\right)+\mathbf{D}_{5}^{(s)} \boldsymbol{u}_{s}+\mathbf{d}_{6}^{(s)} u\left(y_{s}\right),
$$

for the derivative at the interior points. The above expressions can be used to impose the boundary conditions efficiently at discontinuities. In addition, it is straight forward to use basis function composed with maps to handle semiinfinite domains [11].

\section{Conclusion}

The paper addresses an efficient pseudo-spectral method for modelling plasmonic devices. Appropriate conformal maps are used to redefine the basis functions over finite or semi-infinite subdomains, eliminating the need for PMLs. Besides, a precise application of the interface boundary condition is presented. This work can be extended to structures with 2D cross-section or 3D structures having a discontinuity along the $z$ axis.

\section{References}

[1] E. Ozbay, Science 311, 189 (2006)
[2] G. Veronis, S. Fan, Optics Express 15, 1211 (2007)

[3] A.M. Heikal, F.F.K. Hussain, M.F.O. Hameed, S.S. Obayya, Journal of Lightwave Technology 33, 2868 (2015)

[4] M. Koshiba, K. Hayata, M. Suzuki, IEEE transactions on microwave theory and techniques 32, 587 (1984)

[5] K.P. Prokopidis, D.C. Zografopoulos, IEEE Microwave and Wireless Components Letters 24, 659 (2014)

[6] A.M. Said, A. Heikal, N.F. Areed, S. Obayya, IEEE Photonics Journal 8, 1 (2016)

[7] K. Stannigel, M. König, J. Niegemann, K. Busch, Optics Express 17, 14934 (2009)

[8] W. Shin, Ph.D. thesis, Citeseer (2013)

[9] K.S. Atia, A. Heikal, S. Obayya, Optics express 23, 22199 (2015)

[10] L.N. Trefethen, Spectral methods in MATLAB, Vol. 10 (Siam, 2000)

[11] A. Abdrabou, A. Heikal, S. Obayya, Optics express 24, 10495 (2016)

[12] C.C. Huang, Optics express 18, 23711 (2010)

[13] M. Koshiba, Y. Tsuji, Photonics Technology Letters, IEEE 8, 1208 (1996)

[14] H. Rao, R. Scarmozzino, R. Osgood, Photonics Technology Letters, IEEE 11, 830 (1999)

[15] H. El-Refaei, D. Yevick, I. Betty, Photonics Technology Letters, IEEE 12, 389 (2000)

[16] P.L. Ho, Y.Y. Lu, Photonics Technology Letters, IEEE 13, 1316 (2001)

[17] H. Zhang, J. Mu, W.P. Huang, J. Lightwave Technol. 26, 600 (2008)

[18] K.S. Atia, A. Said, A. Heikal, S. Obayya, JOSA B 36, 1402 (2019)

[19] D. Song, L. Yuan, Y.Y. Lu, J. Opt. Soc. Am. A 28, 613 (2011) 